\title{
Localization of Sensor Nodes using Flooding in Wireless Sensor Networks \\ ${ }^{1}$ Basavaraj K Madagouda, ${ }^{2}$ Varsha M Patil, ${ }^{3}$ Pradnya Godse \\ Angadi Institute of Technology \& Management, Belgaum, Karnataka, India basavarajmadagouda@gmail.com
}

\begin{abstract}
The accuracy of localization is a significant criterion to evaluate the practical utility of localization algorithm in wireless sensor networks (WSN). In mostly localization algorithms, one of the main methods to improve localization accuracy is to increase the number of anchor nodes. But the number of anchor nodes is always limited because of the hardware restrict, such as cost, energy consumption and so on. In this paper, we propose a novel which uses forwarding a query message in flooding technique for localization using anchor nodes and once a node localized it acts as virtual anchor node and it helps to localize remaining sensor nodes. It is scheme to increase and upgrade the virtual anchor nodes, while the real number of physical anchors is the same as before.
\end{abstract}

\section{Indexing terms/Keywords}

Flooding, Anchor nodes, Localization, Wireless Sensor network.

\section{Academic Discipline And Sub-Disciplines}

Computer Science and Engineering

\section{SUBJECT CLASSIFICATION}

Localization in Wireless Sensor Networks.

\section{TYPE (METHOD/APPROACH)}

With using on-demand Flooding, finding the loacation of source sensor node using range based algorithm.

\section{Council for Innovative Research}

Peer Review Research Publishing System

Journal: INTERNATIONAL JOURNAL OF COMPUTERS \& TECHNOLOGY

Vol 9, No 3.

editor@cirworld.com

www.cirworld.com, member.cirworld.com 


\section{INTRODUCTION}

Wireless sensor networks (WSNs) [2] are a significant technology attracting considerable research interest. Recent advances in wireless communications and electronics have enabled the development of low-cost, low-power and multifunctional sensors that are small in size and communicate in short distances. Wireless sensor network consists of many sensor nodes which have capabilities of sensing, processing and communication. A specific sensor node is called sink node which collects interested data from sensor nodes.

Wireless Sensor Network (WSN) [3] refers to a group of inexpensive sensors linked by wireless medium, the sensors are provided with sense, computation and Wireless communication capability. WSNs usually are arranged at special scout region, and sense special physics information in the region. Sensor nodes are often deployed by random bestrewing (airplane bestrewing for example), and only a few of nodes assembled with Global Positioning System (GPS [4]) which can capture their position after bestrewing, but most of the nodes cannot acquire such information. Due to its powerful function and low energy cost, the WSN has been widely used in various domains [5], such as national defense and military affair, environment inspection, traffic management, long distance control of dangerous region etc. The localization of sensor nodes has become a hot topic in WSNs. At present, many localization algorithms for sensor networks have been proposed. Most of the WSN includes a small number of anchor nodes, which know their positions beforehand by either using GPS or by manual configuration. Other majority of nodes are unknown position nodes, called unknowns. By using the anchor nodes which knows their location, we can assist the unknowns to be located. But for the existence of various errors, the location precision will be restricted, and some nodes cannot be located.

In this paper, we provide an overview of on-demand techniques, which detects the location of anchor nodes and its location information and other nodes gets localized using the any of the existing localization algorithm techniques can be used for localization of a node [9, 10]. So the improvement of localization precision with lower cost, less energy consumption and less hardware support has become more and more important.

The outline of this paper is as follows. In Section 2 a few localization methods using are being discussed. A proposed technique for on-demand localization of the static sensor nodes is discussed in Section 3. Section 4 shows the simulation and results. Section 5 concludes the paper.

\section{RELATED WORK}

Localization algorithms are divided into two subsections, which are presented by range-based and range-free, according to whether need to measure the range between nodes. For the former, it is required to measure the real ranges between nodes and its neighbors, and use them to calculate the coordinates of nodes. For the latter, instead of real ranges, it uses estimate factors to calculate the coordinates of nodes.

\subsection{Range-based Localization Algorithms}

Time of Arrival (TOA) [11] technology is used to measure range through signal propagation time. The most typical application of TOA technology is GPS. The Time Difference of Arrival (TDOA) [9] technique has been proposed as a localization solution by using two different signals (such as ultrasound and RF). By registering the time difference of signal arrival, it uses the propagating velocity to translate the time difference into distance.

Angle of Arrival (AOA) [13] technology has been proposed to utilize array antennas or combined multi-receivers to obtain the propagation direction of signal. Based on the directions, it gets the range between neighbors.

Received Signal Strength Indicator (RSSI) [9] technology is used to calculate range via signal strength with theoretical or empirical models. The main error source of this technology is the complexity of signal propagation models caused by environment influence. The combination of RSSI and other location algorithm will make a better scheme.

\subsection{Range-free Localization Algorithms}

Centroid algorithm [15] is a simple range-free localization algorithm. The node receives signals of anchors in its communication area and makes its coordinates as the centroid of these anchors. DV-Hop algorithm [16] is the most basic scheme, and it first employs a classical distance vector exchange so that all nodes in the network get distances, in hops, to the anchors. Once an anchor gets distances to other anchors, it estimates an average size for one hop, which is then deployed as a correction to the entire network. When receiving the correction, an arbitrary node may then have estimate distances to anchors, in meters, which can be used to estimate the coordinates of nodes. Amorphous algorithm [16] is similar to DV-hop, but it assumes to know the network density in advance, and uses offline hop-distance estimations. In APIT [17], the network area is isolated into some triangular regions by anchors. A node's presence inside or outside of these triangular regions allows a node to narrow down the area in which it can potentially reside. 


\section{NETWORK MODEL AND DIFINITIONS}

\subsection{WSN model}

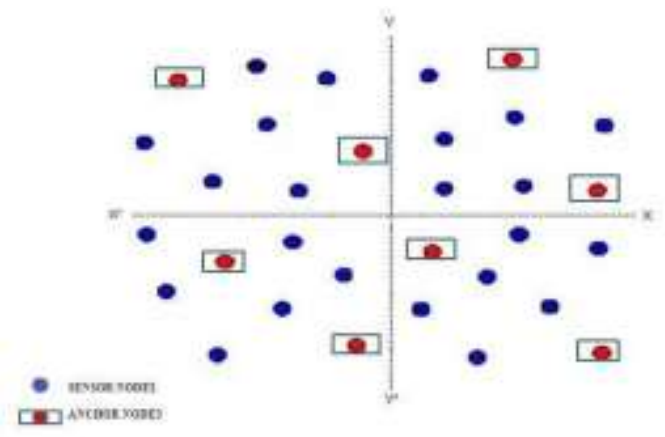

Figure1: A typical Wireless Sensor Network.

Consider a WSN as shown in figure 1 which consists of several nodes scattered randomly. The nodes are of two types: anchor nodes and sensor nodes. Anchor nodes are the nodes which have priori information about their location before deployment. These nodes are equipped with a more powerful GPS system and which localizes with ease. The network model consists of several sensor nodes and few anchor nodes populated. After localization, these nodes act as base stations and send radio waves incessantly which are of low range ranging between 50-100 meters. Each anchor node has a specific ID stored in its memory which is sent along with the radio signals. On the other hand sensor nodes are the nodes whose location is unknown. The sensor nodes are GPS less nodes which localize themselves with the help of the anchors in their proximity or with the help of the other neighboring sensor nodes. This model has very few anchor nodes and many sensor nodes which are light weighted and less expensive than the anchor nodes.

Query message - A message sent to a node to find out the value of one of the node's properties. The properties are the $x$ co-ordinate and y co-ordinate of the node.

Virtual Anchor Node - The sensor nodes which localize using the location of anchor nodes are called Virtual anchor node (VAN).

A data table - Each sensor node contain a data table which stores the information about location of each anchor node. Fields of data table includes anchor node ID, X co-ordinate and y co-ordinate of the anchor node and the distance from each anchor node with its hop count.

TTL [Time to Live] - The amount of time required to send and receive a request and response message is known as TTL. To find TTL, we consider parameters $s$ and $d$ where $s$ denotes speed of network and $d$ is the hop count distance, $\mathrm{TTL}=\mathrm{d} / \mathrm{s}$.

Hop count limit $[\mathrm{HC}]$ - It specifies a limit on the number of hops a query message is allowed to broadcast in the network.
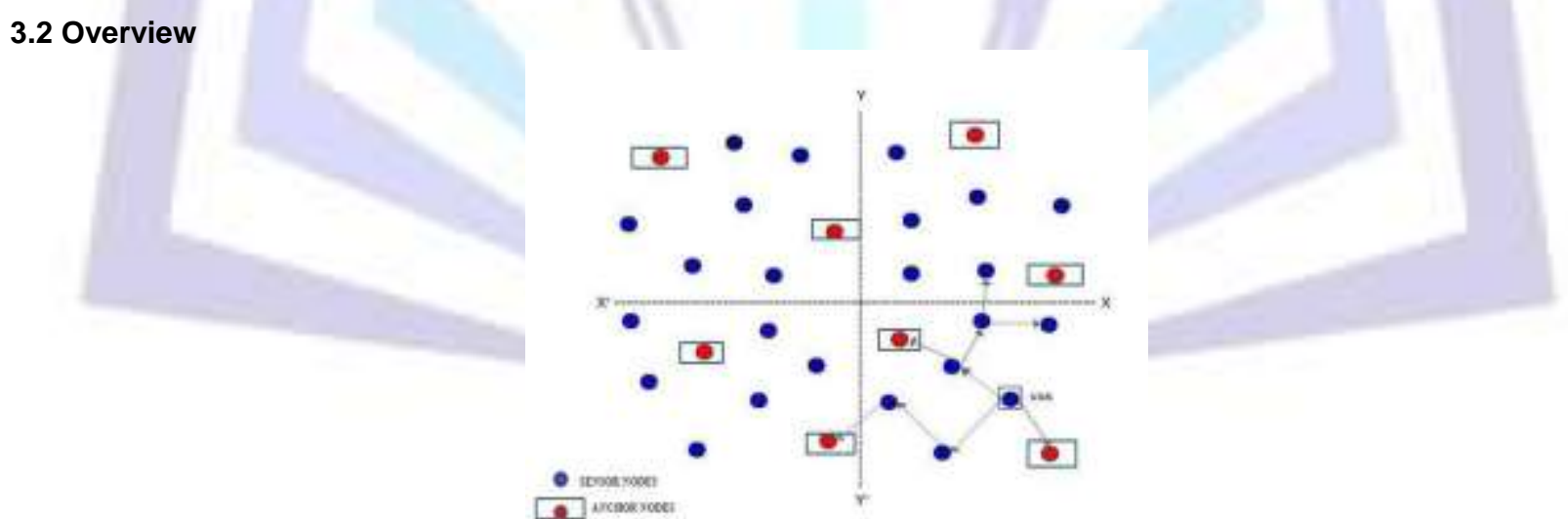

Figure2: Flooding query message for localizing a node.

In figure 2, some nodes have their location information and other nodes don't have their location information. To finding location of sensor nodes is known as localization. To find location of sensor nodes, broadcasts a query message to its neighboring nodes for location information of anchor nodes. If any anchor node is in its broadcasting range it replies to that query message and using any range based free algorithm, sensor node gets localized. Sensor nodes which get localized stores all anchor node information in its data table as shown in Table.1 and it acts as a virtual anchor node for the other which are not localized. Reminder set of sensor nodes which do not have localization inform, once again broadcast a query message. If any Virtual anchor node gets a query message then it replies all anchor nodes information which has stored in its table. A node localized using anchor nodes information and it acts as VAN for other sensor nodes.

Sensor node which has been localized stores all information about anchor nodes with the hop distance in its table. Any virtual anchor node receives a query message sent by other sensor nodes to get information about the anchor nodes. Virtual anchor node sends all anchor nodes' information which is stored in its data table with adding the hop distance 
(between source node to VAN) to each node in its data table. The sensor node gets localized using the information from the virtual anchor node and it also stores all anchor node information in its data table.

TABLE 1. STRUCTURE OF DATA TABLE

\begin{tabular}{cccc}
\hline $\begin{array}{c}\text { Anchor } \\
\text { Node ID }\end{array}$ & $\begin{array}{c}\text { X co- } \\
\text { ordinates }\end{array}$ & $\begin{array}{c}\text { Y co- } \\
\text { ordinates }\end{array}$ & $\begin{array}{c}\text { Hop } \\
\text { Distance (d) }\end{array}$ \\
\hline 1 & 36.7 & 15.9 & 2 \\
3 & 65.2 & 32.1 & 1 \\
4 & 45.4 & 70.3 & 3 \\
. &. &. &. \\
N & 83.39 & 9.87 & 10 \\
\hline
\end{tabular}

\subsection{Localization scheme: Two phases}

\subsubsection{Localization using flooding technique}

In wireless sensor network the sensor nodes localize using the information about the anchor nodes, broadcasting the query message in all directions to its neighbor nodes in its transmission range as shown in figure 3 . Sensor node sends the query message using flooding scheme, other nodes receive same query message multiple times from different paths.

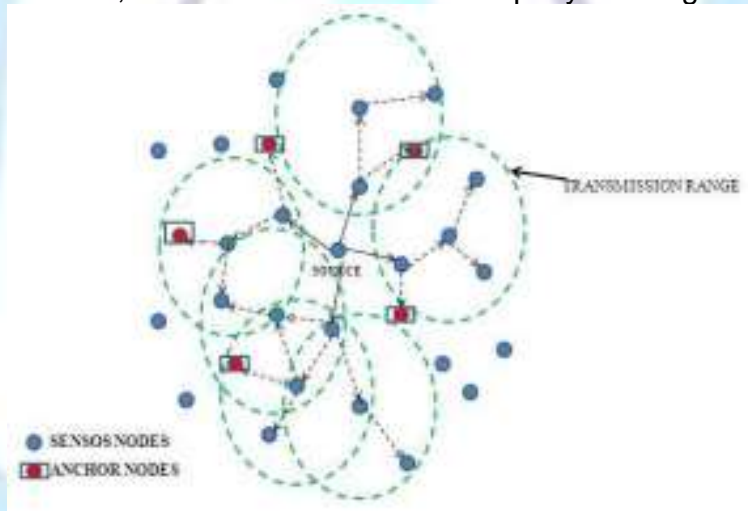

Figure 3: Localization using flooding technique

The amount of consumption energy to broadcast is given by:

Etotal $=\left(\text { Erx }{ }^{*} \text { the average number of neighbors }+E t x\right)^{*} \mathrm{~N}$

As equation (1) [7] means, the total energy directly depends on the number of all nodes $(\mathrm{N})$ and the average number of neighbors. So, the total energy consumed for one broadcast is big so that it can rapidly reduce network's lifetime. The life time of nodes become less and also decreases the network life time.

\subsubsection{Localization using Virtual Anchor Nodes}

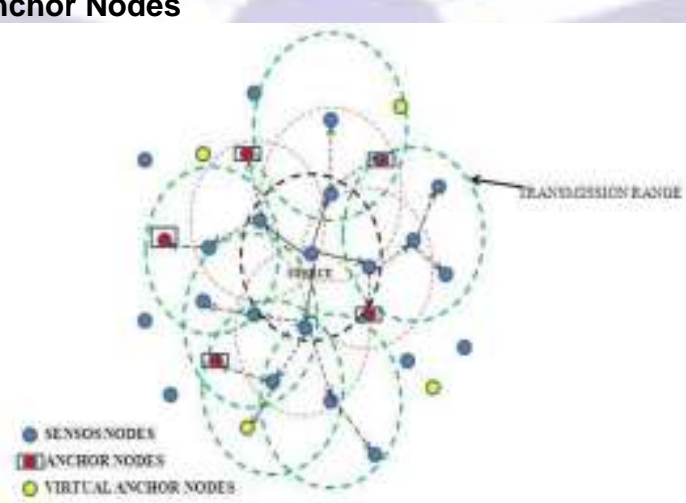

Figure 4: Localization using Virtual Anchor Nodes

Using On-demand technique the sensor nodes obtain the information about anchor nodes. Sensor node broadcasts a query message to its neighbor nodes which are in its radio frequency range by setting TTL and the hop count. Each sensor node contains a memory to store the information about the query message received from the sensor nodes. Once 
it receives the message, it decreases the hop count which is set by the source node in network. The sensor node which receives a query message broadcasts the same message to its neighbor nodes which are in its radio frequency range. The received node in turn sends the query message to its neighbor nodes. Every node checks its memory when a new message is received and it discards if the same message is received twice. In two conditions the sensor node does not get localized. First condition is if the hop count value becomes zero and the source node does not get any information about minimum three anchor nodes information. Then the source node re-broadcasts the query message by increasing the hop count value. Second condition is when the Time to live becomes zero then the broadcasting of query message will be stopped. When an anchor node receives the query message, it sends its location information to the node from which it received in the same path. After receiving information about anchor nodes, sensor node stores all anchor nodes information in its data table. If a sensor node get minimum three anchor nodes information, then it calculates its location using information about anchor nodes and also it stores forwarding time (tF) and backward time (tB). When a sensor node gets localized it acts as Virtual Anchor Node (VAN). Upon receiving query message by virtual anchor node (VAN), it renders all anchor node information from its table to sensor node. Sensor node updates the hop count values and stores all information in its data table. A sensor node can localize using anchor node information from VAN. With this mechanism we can reduce the communication cost of sensor nodes, energy consumption of sensor nodes and also minimize the network lifetime.

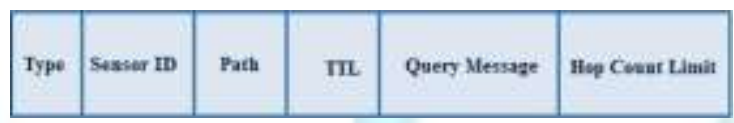

Figure 5: Format of request packets

The request packet format of query message send by the sensor node is shown figure 5 . The fields of packet are type, sensor node ID, path, TTL (time to leave), query message and hop counts. First field Type indicates that the message is request message or response message. If Type value is 0 , then its request message. Also the packet contains the sensor node ID which has broadcast a query message, TTL value. Path field contains the path in which the query message is sent. The hop count limit is value to broadcast the query message from a source node to it other nodes.

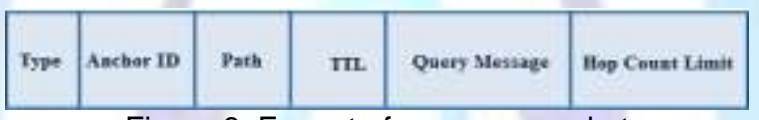

Figure 6: Format of response packet

The response packet format sent by the anchor node is shown in figure 6 . The fields of packet are type, sensor node ID, path, TTL, query message and hop counts. If Type value is 1 , then its response message and the packet contain the anchor node ID which has responding a query message, TTL value. The anchor node sends its information in same path in which path it received that query message. The hop count is value to find the distance from source node to anchor node.

\subsubsection{Trilateral positioning method}

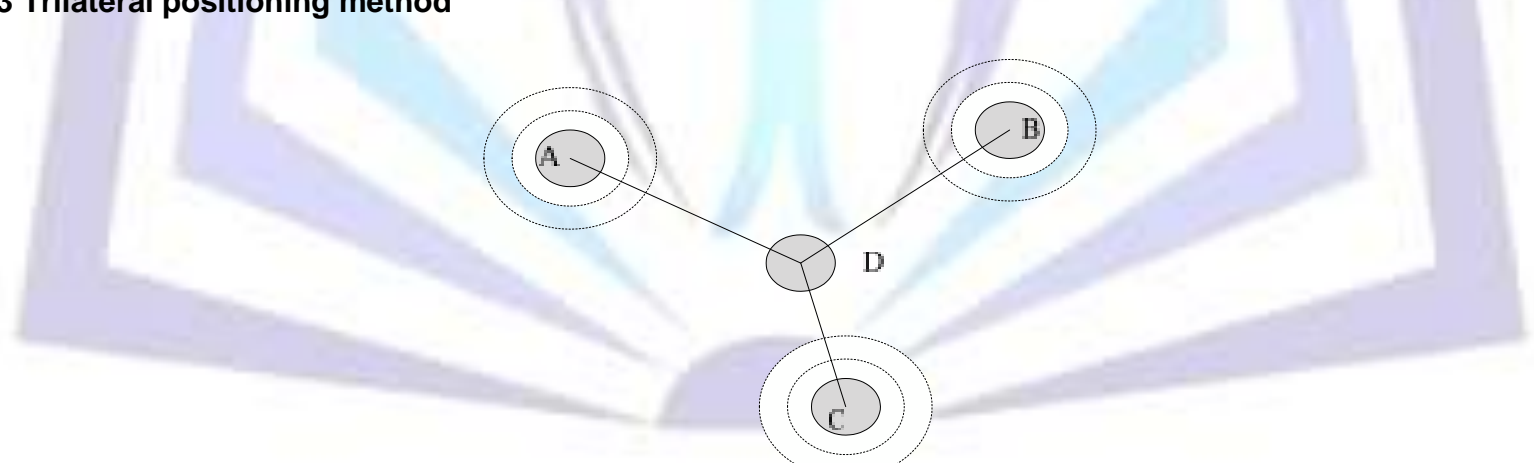

Figure7: The principle of trilateral positioning method.

If we have the coordinates of three anchor node $A\left(x_{A}, y_{B}\right), B\left(x_{B}, y_{B}\right), C\left(x_{C}, y_{C}\right)$ and unknown node $D(x, y), d_{A}, d_{B}, d_{C}$ are the distance from the unknown node to the three anchor nodes as shown in figure7. The calculation formula is as follows [18]:

$$
\begin{aligned}
& d_{A}^{2}=\left(X-X_{A}\right)^{2}+\left(Y-Y_{A}\right)^{2} \\
& d_{B}^{2}=\left(X-X_{B}\right)^{2}+\left(Y-Y_{B}\right)^{2} \\
& d_{C}^{2}=\left(X-X_{C}\right)^{2}+\left(Y-Y_{C}\right)^{2}
\end{aligned}
$$

We can calculate the coordinates of the source node with using below equation (2)

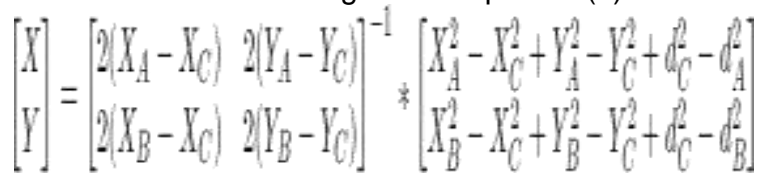


Because the three circles will not intersect at one point, $d_{A}, d_{B}, d_{C}$ which are measured in the trilateral localization algorithm have measurement error, then the calculated coordinates of the unknown node and the actual coordinates for comparison, the error will be large. To reduce the impact of distance error in the localization accuracy, multilateral great likelihood estimation method can be adopted.

\subsubsection{Localization of static sensor nodes}

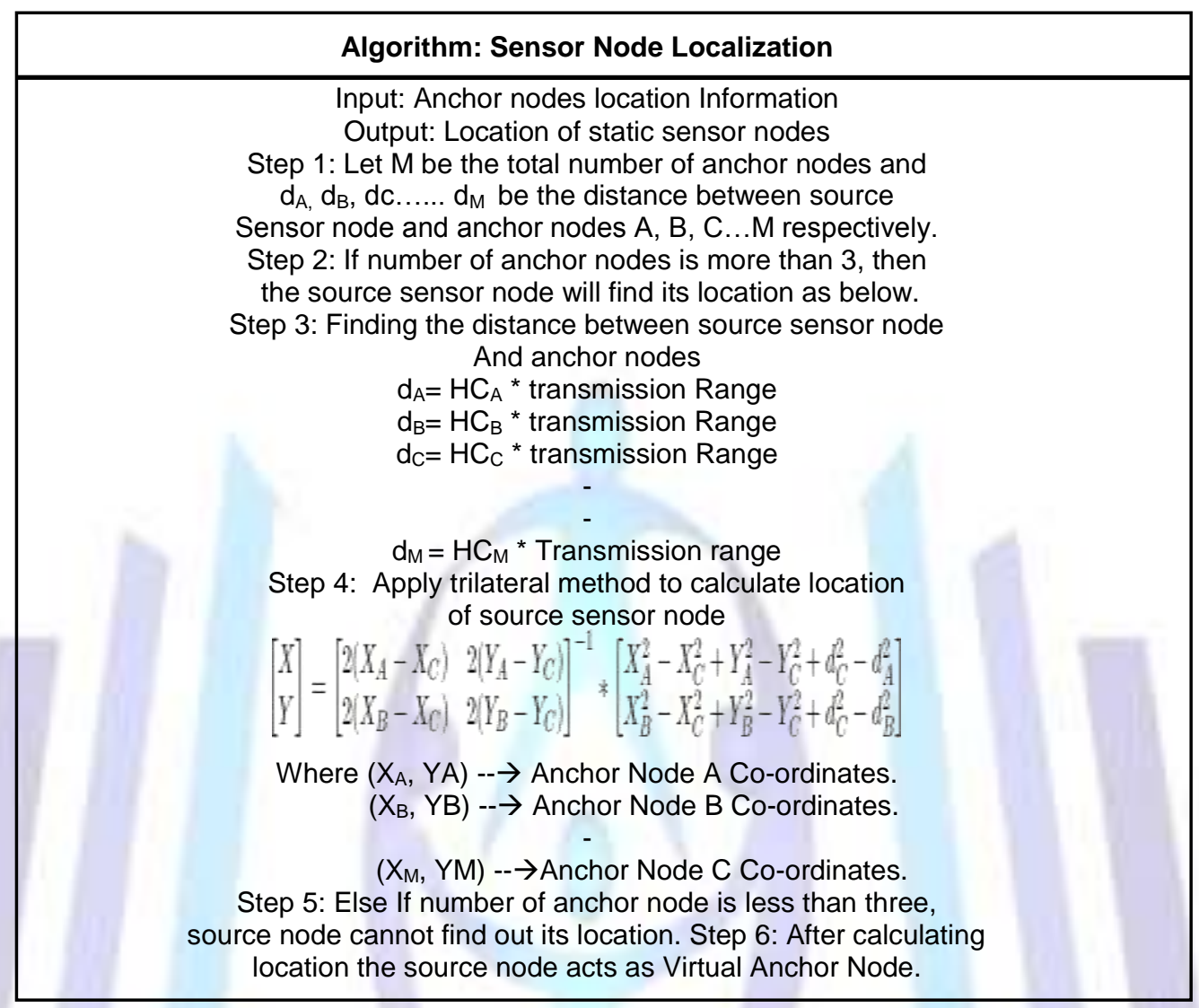

\section{SIMULATION AND RESULTS}

The WSN is simulated using Microsoft Visual Studio/ C \#. The simulated network model consists of 50 sensor nodes of which 15 are anchor nodes populated over a 100X100 grid area. The nodes are distributed randomly and uniformly over the deployment area.

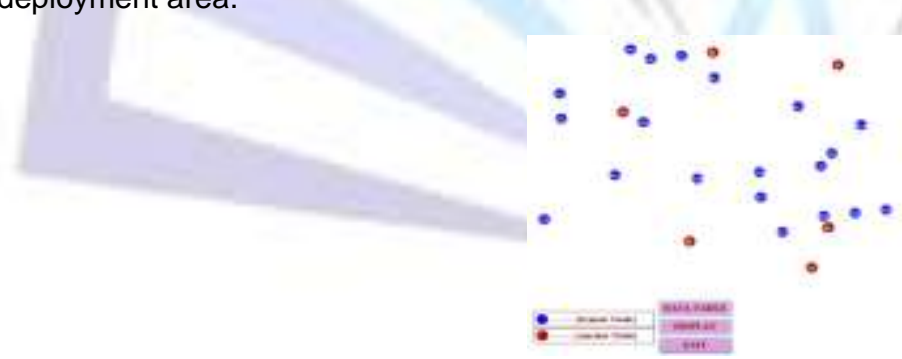

Figure 8:Shows the deployment of sensor nodes and anchor nodes.

A scatter plot graph depicting the deployed nodes is shown in figure 8 . The list boxes are provided to store the path and hop count which is limited to three of the source node from other nodes. Data Table is to view number of anchor nodes with its range of source node. The list boxes are provided to store the path of the source node from other nodes. Display is for displaying the all anchor nodes location. 


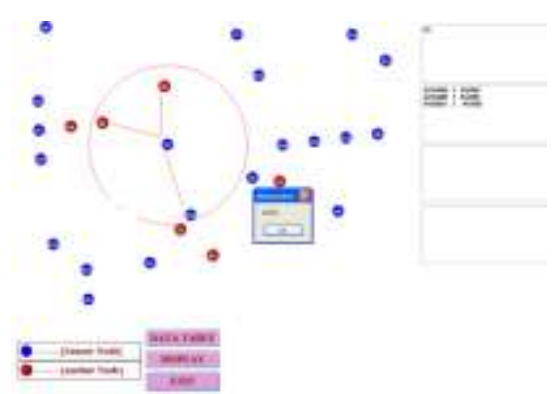

Figure 9:Shows the sending of query message in first hop.

In figure 9, When user clicks on any of the node it acts as source node and starts flooding the message to its neighboring nodes. When the source node sends the message to the neighboring nodes the Hop Count will be set to 1 and the path information is displayed in the list boxes. If the source node gets any anchor node in first hop the anchor node sends its location to the source node via same path.

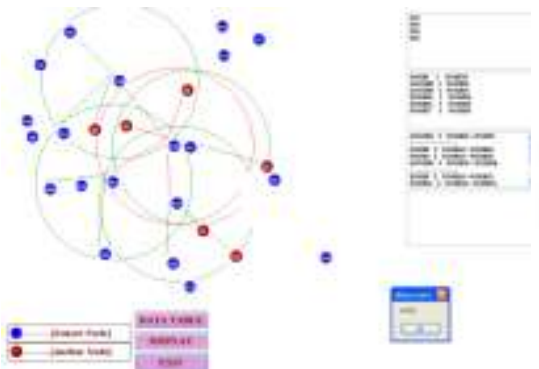

Figure 10:Shows the sending of query message in second hop.

Once the Hop Count of sensor node becomes 1 then neighboring sensor nodes, further flood the messages to its neighboring nodes and the Hop Count will be set to 2 of nodes which come in its transmission range as shown in above figure10. If there is any anchor node then it will send its location to the source node via same path.

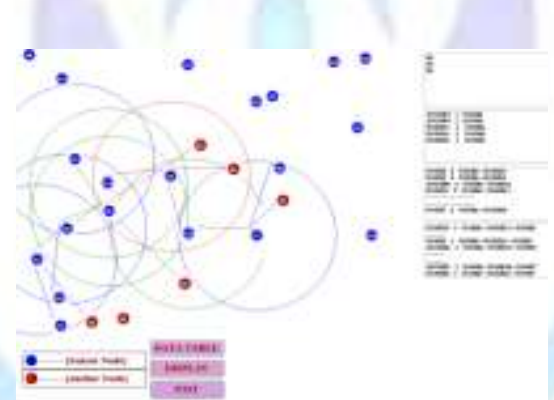

Figure11: Shows the sending of query message in third hop.

Once the Hop Count of sensor node becomes 2 then it will further flood the messages to its neighboring nodes and Hop Count will be set to 3 of nodes which come in its transmission range. If there is any anchor node then it will send its location to the source node via same path as shown in above figure 11.

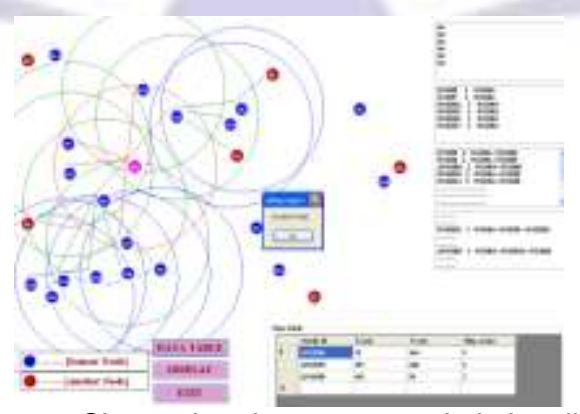

Figure 12:Shows that the source node is localized.

In figure 12, once the source node gets the location of minimum three anchor nodes it stores it in a data table and when user clicks on data table button the source node finds its location and gets localized. 


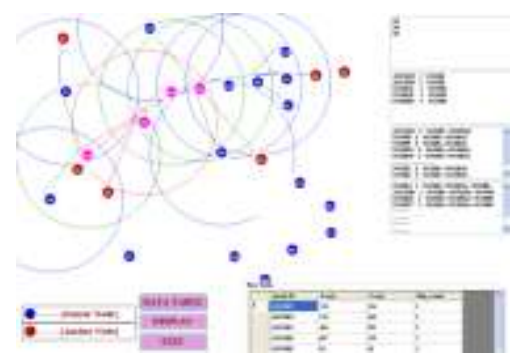

Figure 13:Shows that the source node acts as Virtual Anchor Node.

In figure 13, Once the source node gets localized, if the nodes which come in its range send the request message to source node then the source node sends the anchor nodes information to the node which is sending request and it help localize the nodes which come in its range.

The following metrics are used to measure the performance of the proposed scheme: localization error, localization ratio and energy efficiency ratio. Localization error defines the amount of error involved in the location information of the dislocated node. Localization ratio is defined as the ratio of the number of nodes which successfully get localized to the number of nodes deployed in network. Energy efficiency ratio is defined as the ratio of the total energy is used by nodes to get localized to the total amount of energy in the network.

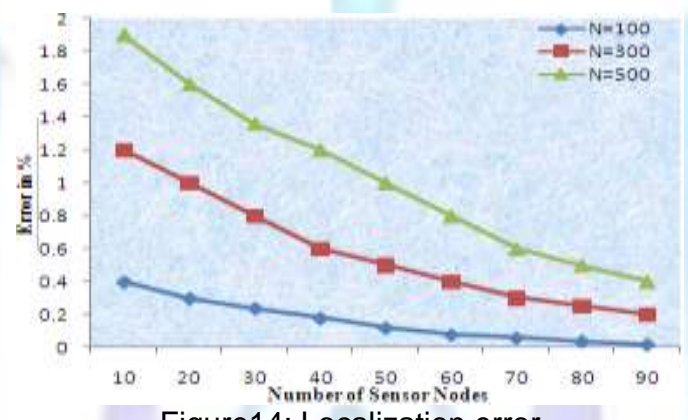

Figure14: Localization error

Figure 14 shows a graph plotted for localization error. The $x$-axis represents the number of nodes dislocated and the $y$ axis represents the localization error in percentage. It depicts that, when the total number of nodes deployed $(\mathrm{N})$ in an application increases the error decreases. The localization error [18] is calculated using

$$
\text { Localization Error }=\frac{\sum\left(X_{e}-X_{l}\right)+\left(Y_{e}-Y_{l}\right)}{\text { Numberof Sensor Nodes }}
$$

Where $\left(\mathrm{X}_{\mathrm{e}}, \mathrm{Y}_{\mathrm{e}}\right)$ are the estimated location of sensor node and $(\mathrm{XI}, \mathrm{YI})$ is the true location of sensor node.

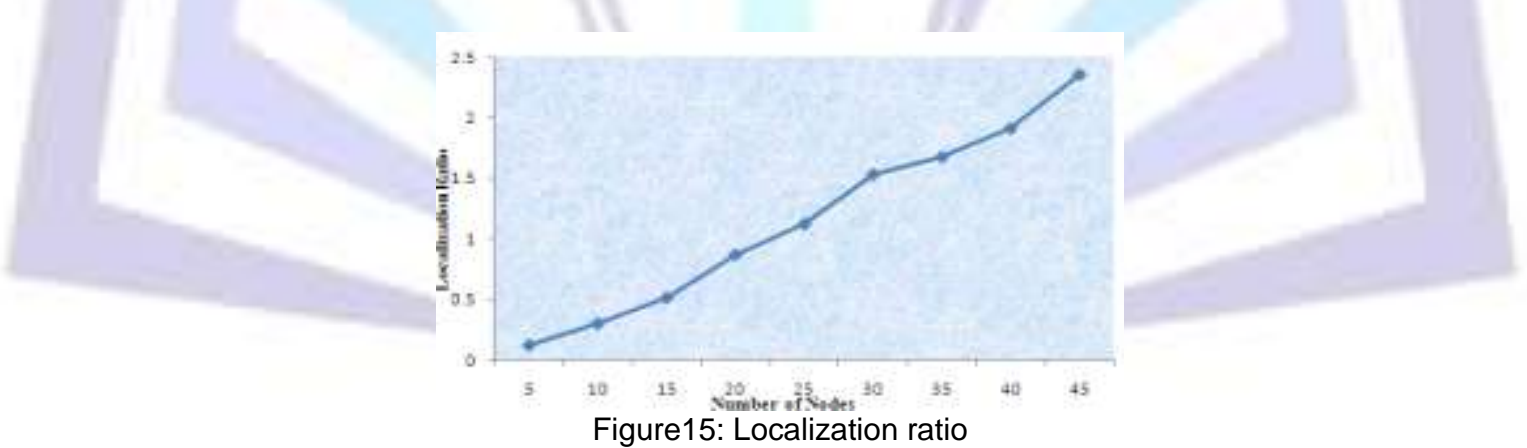

Figure 15 shows a graph plotting the number of nodes in the $x$-axis and the localization ratio in the $y$-axis. It shows that the ratio of the energy consumed by the nodes getting localized with the total energy in the network in the application.

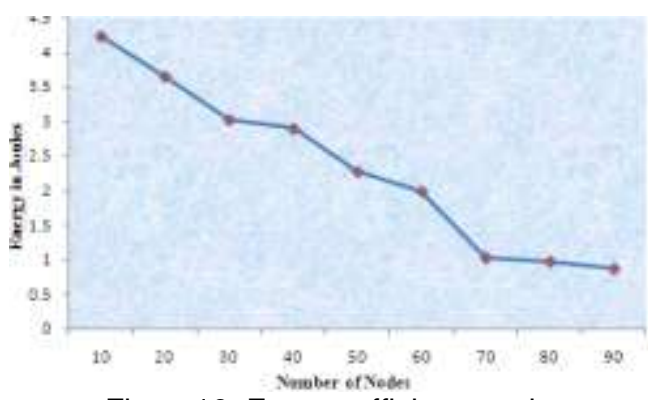

Figure16: Energy efficiency ratio 
Figure 16 shows a graph plotting the number of nodes in the x-axis and the energy in the $y$-axis. It shows that the ratio of the energy consumed by the nodes getting localized with the total energy in the network in the application.

\section{Conclusion}

Precision is an important criterion for any wireless sensor network to work adeptly. In the paper we show an on- demand flooding technique to reduce the number of broadcasts and localizing other sensor nodes with lower cost, less energy consumption and less hardware support. Also our technique efficiently identifies the location of anchor nodes and virtual anchor nodes information to localize sensor nodes with minimum number of broadcasts. Then it helps the node to trigger the localization algorithm and gets localized on demand. Thus the location information of the sensor nodes is accurate and the WSN operates without any flaw.

\section{References}

[1] Basavaraj K Madagouda, Sumathi R, A. H. Shanthakumara," Localization of Sensor Nodes using Flooding in Wireless Sensor Networks", in ObCom 2011, Part I, CCIS 269, pp. 637-646, 2012.@ Springer-Verlag Berlin Heidelberg 2012.

[2] C.-Y. Chong, S. Kumar, Sensor networks: evolution, opportunities, and challenges, Proceedings of the IEEE 91(8) (2003) 1247-1256.

[3] Vidyasagar Potdar, Atif Sharif, Elizabeth Chang, "Wireless Sensor Networks: A Survey", International Conference on Advanced Information Networking and Applications Workshops, 2009.

[4] B. Hofmann-Wellenhof, H. Lichtenegger, and J. Collins, "Global Positioning System: Theory and Practice", SpringerVerlag, 4th edition, 1997.

[5] Th. Arampatzis, J. Lygeros, S. Manesis, "A Survey of Applications of Wireless Sensors and Wireless Sensor Networks", Proceedings of the 13th Mediterranean Conference on Control and Automation Limassol, Cyprus, 2005.

[6] Pengxi Liu, Xinming Zhang, Shuang Tian, Zhiwei Zhao, Peng Sun Department of Computer Science and Technology University of Science and Technology of China "A Novel Virtual Anchor Node-based Localization Algorithm for Wireless Sensor Networks", Proceedings of the Sixth International Conference on Networking, 2007.

[7] Ning Yu, Jiangwen Wan, "On Demand Localization Algorithm for Wireless Sensor Networks".

[8] Jeongho Son, and Tae-Young Byun, "A Routing Scheme with Limited Flooding for Wireless Sensor Networks", International Journal of Future Generation Communication and Networking Vol. 3, No. 3, September 2010

[9] Cesare Alippi, Giovanni Vanini, "A RSSI-based and calibrated centralized localization technique forWireless Sensor Networks", Proceedings of the Fourth Annual IEEE International Conference on Pervasive Computing and Communications Workshops (PERCOMW'06), 2006.

[10]R. Peng, M. L. Sichitiu, Angle of Arrival Localization for Wireless Sensor Networks, Third Annual IEEE Communications Society Conference on Sensor and Ad Hoc Communications and Networks, (Reston, VA), Sep. 2006.

[11]K. Lorincz and M. Welsh, "MoteTrack: A robust, decentralized approach to RF-based location tracking," T. Strang and C. Linnhoff- Popien, editors, Location- and Context- Awareness: First International Workshop, German, vol. 3479 of Lecture.

[12] Hao Ni, Guangliang Ren, and Yilin Chang, "A TDOA Location Scheme in OFDM Based WMANs", IEEE Transactions on Consumer Electronics, 2008.

[13]Binwei Deng, Guangming Huang, Lei Zhang and Hao Liu, "Improved Centroid Localization Algorithm in WSNs", Proceedings of 3rd International Conference on Intelligent System and Knowledge Engineering, 2008.

[14]Byeong-Tae Lee, Sunwoo Kim, "Scalable DV-Hop Localization for Wireless Sensor Networks", Proceedings of APCC2008, 2008.

[15] R. Nagpal, "Organizing a global coordinate system from local information- on an amorphous computer," A.I. Memo 1666, MIT A.I. Laboratory, August 1999.

[16]Ji zeng Wang, Hongxu Jin, "Improvement on APIT Localization Algorithms for Wireless Sensor Networks", Proceedings of International Conference on Networks Security, Wireless Communications and Trusted Computing, 2009.

[17]Vibha Yadav, Manas Kumar Mishra, A.K. Sngh and M. M. Gore, "LOCALIZATION SCHEME FOR THREE DIMENSIONAL WIRELESS SENSOR NETWORKS USING GPS ENABLED MOBILE SENSOR NODES" Department of Computer Science \& Engineering, Motilal Nehru National Institute of Technology, Allahabad, INDIA, International Journal of Next-Generation Networks (IJNGN),Vol.1, No.1, December 2009.

[18] Xiaohui Chen, Canfeng Gong and Jiangbo Min, "A Node Localization Algorithm for Wireless Sensor Networks based on Particle Swarm Algorithm”, JOURNAL OF NETWORKS, VOL. 7, NO. 11, NOVEMBER 2012 